Artigo Original

Original Article

\title{
Sprints como atividade condicionante para aumentar o desempenho no salto em distância: um estudo experimental
}

\section{Sprints as Conditioning Activity to Improve the Long Jump Performance: An Experimental Study}

\author{
Diego de Alcantara Borba ${ }^{\S 1} \mathrm{MS}$; Guilherme de Castro Lopes ; Victor Simões Campos D’al Ferro \\ Recebido em: 29 de maio de 2019. Aceito em: 10 de junho de 2019. \\ Publicado online em: 31 de julho de 2019.
}

\section{Resumo}

Introdução: Atividades condicionantes com intensidades máximas são utilizadas para provocar aumento no desempenho esportivo por meio da potencialização pós-ativação. Porém, há poucos estudos com indivíduos não atletas.

Objetivo: Avaliar o efeito de dois sprints de curta duração como atividade condicionante sobre o desempenho do salto em distância. Métodos: Estudo do tipo experimental, com amostra por conveniência, do qual participaram 12 voluntários do sexo masculino (24 \pm 6 anos), não treinados. Os participantes foram expostos a duas situações experimentais, de modo aleatório, com intervalo de cinco minutos entre ambas: 1) Controle: seis saltos em distância; 2) C10s: Dois sprints de 10 segundos seguidos de seis saltos em distância após com cinco minutos de repouso entre os sprints e os saltos. Foi utilizado o teste $t$ de Student pareado para comparar as situações. A resposta individual ao protocolo de potencialização foi analisada por meio do erro típico. 0 índice de significância foi menor que $5 \%$.

Resultados: A distância do salto foi maior na situação C10s comparado ao controle. A análise individual mostrou que $50 \%$ dos participantes responderam positivamente, $25 \%$ negativamente e

\begin{tabular}{|l|}
\hline Pontos-Chave Destaque \\
- Há poucos estudos sobre a \\
aplicação de atividades \\
condicionantes (AC) para \\
promover potencialização pós- \\
ativação (PPA) em indivíduos \\
não treinados. \\
- Em $50 \%$ dos participantes, \\
houve melhora no desempenho \\
no salto em distância. \\
- Em $25 \%$, houve piora no \\
desempenho e em $25 \%$ nenhum \\
efeito.
\end{tabular}
$25 \%$ foram indiferentes ao protocolo de potencialização.

Conclusão: Os sprints utilizados no presente estudo foram capazes de desencadear a potencialização pósativação e consequente aumento no desempenho do salto em distância em sujeitos não treinados.

Palavras-chave: atividade preparatória, força, atletismo.

\begin{abstract}
Introduction: Maximum intensities conditioning activities are used to increase sports performance through post-activation potentiation. However, there are few studies with non-athletes.

Objective: To evaluate the effect of two sprints of short duration as conditioning activity on the long jump performance.

Methods: A study of the experimental type, with convenience sample, in which 12 male volunteers (24 \pm 6 years old), untrained, participated. Participants were exposed to two experimental situations, randomly, with a five-
\end{abstract}

\footnotetext{
$\S$ Autor correspondente: Diego de Alcantara Borba - e-mail: diegoalcantara1@gmail.com Afiliações: ${ }^{1}$ Universidade do Estado de Minas Gerais, Departamento de Ciências do Movimento Humano, Unidade de Ibirité, MG; 2Universidade de Itaúna, Curso de Educação Física, Itaúna, MG.
} 
minute interval between the two: 1) Control: six distance jumps; 2) C10s: Two sprints of 10 seconds followed by six distance jumps after five minutes of rest between sprints and jumps. The paired Student t test was used to compare the situations. The individual response to the potentiation protocol was analyzed by means of the typical error. The significance level was less than $5 \%$.

Results: The distance of the jump was greater in the C10s situation compared to the control. The individual analysis showed that $50 \%$ of the participants responded positively, 25\% negatively and for $25 \%$ protocol potentiation showed no effect.

Conclusion: The sprints used in the present study were able to trigger the post-activation potentiation and consequently improved the long jump performance in untrained individuals.

Keywords: preparatory activity, strength, athletics.

\section{Keypoints}

- There are few studies on the application of conditioning activities (CA) to promote postactivation potentiation (PPA) in untrained individuals.

- In $50 \%$ of the participants, there was improvement in the performance in the distance jump.

- In 25\%, there was worse performance and $25 \%$ no effect.

\section{Sprints como atividade condicionante para aumentar o desempenho no salto em distância: um estudo experimental}

\section{Introdução}

O salto em distância é caracterizado por grande manifestação de força, no qual o atleta deve gerara a maior força explosiva possível no momento da impulsão sobre a tábua. No cotidiano do treinamento e competição a capacidade de gerar força muscular pode ser melhorada agudamente pela realização de atividades condicionantes a fim de desencadear a potencialização pósativação(1,2). A potencialização pós-ativação (PPA) é um mecanismo neuromuscular responsável em aumentar (potencializar) o desempenho físico e esportivo(1). Segundo Sale(2), a potencialização é provocada por uma atividade condicionante (AC) realizada em intensidades máximas ou próximas da máxima, desencadeada por mecanismos fisiológicos centrais e musculares relacionados ao aumento na velocidade de condução dos impulsos nervosos ao músculo e maior ativação das unidades motoras do tipo $\operatorname{IIx}(3,4)$ envolvendo a ativação da cadeia leve de miosina e o aumento da atividade do $\mathrm{Ca} 2+(5)$. $\mathrm{O}$ resultado dessa estimulação seria a melhor conexão entre os filamentos contráteis, favorecendo melhora na capacidade de gerar tensão, ou seja, a PPA(2).

A literatura mostra que vários protocolos de exercícios resistidos $(1,6)$ e de saltos $(7,8)$ têm sido utilizados como AC para desencadear a
PPA, em diversas modalidades esportivas. Os estudos identificados sobre o tema foram realizados em indivíduos treinados em força ou atletas das respectivas atividades principais. Segundo Chiu et al.(9) a PPA é mais evidente neste tipo de população. Isso se deve a melhor capacidade de atletas e sujeitos treinados em força e potência muscular em recrutar melhor as unidades motoras do tipo IIx, que são mais susceptíveis aos mecanismos de PPA(10). Apenas um estudo investigou os efeitos da AC na PPA em sujeitos destreinados. Borba et al.(11) mostraram melhora da distância do arremesso do peso em sujeitos destreinados após sete minutos da realização do exercício no supino reto.

O presente estudo teve por objetivo avaliar o efeito de uma AC em corrida de velocidade sobre o desempenho do salto em distância, em sujeitos não treinados em força ou com experiência nesta modalidade. A hipótese é a de que a distância do salto aumento após a realização da $\mathrm{AC}$ devido à manifestação da PPA.

\section{Métodos}

\section{Desenho de estudo e amostra}

Estudo do tipo experimental, com amostra por conveniência, do qual participaram 12 voluntários do sexo masculino ( $24 \pm 6$ anos; $1,78 \pm 0,08 \mathrm{~m} ; 73,2 \pm 9,1 \mathrm{~kg} ; 10,4 \pm 4,4 \% \mathrm{G})$. 
Todos os voluntários possuíam experiência em esportes, porém, sem experiência com o salto em distância e não praticavam treinamento de força há pelo menos dois anos.

Os critérios de inclusão foram: 1) indivíduos entre 18 e 35 anos; 2) indivíduos que, ao responderem o Par-Q (Physical Activity Readiness Questionnaire), não apresentassem objeções. Os critérios de exclusão foram: 1) apresentar alguma limitação física quanto à realização das tarefas de corrida e saltos antes ou durante o estudo; 2) Apresentar ou adquirir qualquer tipo de doença ou infecção antes e durante a intervenção.

O presente estudo foi aprovado pelo Comitê de Ética em Pesquisa da Universidade do Estado de Minas Gerais (parecer 1.306.982). Os procedimentos respeitaram a resolução de pesquisa com seres humanos do Conselho Nacional de Saúde (466/2012). Os participantes foram pessoalmente convidados pelos pesquisadores. Ao concordarem em participar, os voluntários assinaram o Termo de Consentimento Livre e Esclarecido e responderam ao questionário de prontidão para atividade física Par-Q antes de participarem do estudo.

\section{Medidas}

A estatura e a massa corporal foram mensuradas utilizando balança com estadiômetro (Filizola ${ }^{\circledR}$, Brasil). Para estimar o percentual de gordura foi utilizado o como referência para o cálculo o método das dobras cutâneas e a equação $\left[1.112-0.00043499 \times\left(\sum\right.\right.$ 7 dobras $)+0.00000055 \times\left(\sum 7\right.$ dobras $) 2-$ $0.00028826 \mathrm{x}$ (idade)] sugerido por Jackson e Pollock(12). A Percepção Subjetiva do Esforço (PSE) foi estimada utilizando a Escala de seis a 20 itens sugerida por Borg e Noble(13). O participante indicou qual número da escala corresponderia sua percepção momentânea da dificuldade do exercício. Antes da $\mathrm{AC}$ o pesquisador orientou $\mathrm{o}$ voluntário a dar nota "6" para sua PSE de ausência de esforço ou dificuldade e nota 20 como o máximo esforço físico ou dificuldade já realizado por ele em algum esforço físico anterior. A PSE foi então apresentada para o voluntário logo após a $\mathrm{AC}$ e cinco minutos após a $\mathrm{AC}$, momento que corresponde o início da atividade principal (saltos). A distância do salto foi mensurada em metros e com uso de fita métrica com precisão de milímetros, posicionada no início na borda posterior da tábua até a marca mais próxima dos pés a tábua de impulsão (ponto do salto).

A duração da $\mathrm{AC}$ foi registrada com uso de cronômetro no qual este foi acionado no comando de "já" e cravado após comunicação do término do tempo determinado para a AC.

\section{Procedimentos e atividade condicionante}

$\mathrm{Na}$ primeira visita foram realizadas as medidas antropométricas e logo em seguida o voluntário realizou a familiarização da $\mathrm{AC}$, ou seja, o salto em distância na caixa de areia. A familiarização consistiu em três séries de seis saltos em distância com intervalo de dois minutos a cada três saltos. A técnica do salto foi padronizada do seguinte modo: o participante se posicionava com um dos pés sobre a tábua de impulsão, o outro pé atrás da linha do quadril e o tronco e joelhos levemente flexionados. A impulsão (salto) então foi realizada projetando a perna de trás a frente com o joelho flexionado juntamente com a extensão do joelho da perna da frente. Durante a impulsão foi permitido o uso do movimento de balanço para trás e para frente dos braços. A aterrissagem foi realizada com ambos os pés ao mesmo tempo. A cada tentativa o pé sobre a tábua foi alternado.

$\mathrm{Na}$ segunda visita, 48 horas após a familiarização, os participantes realizaram de modo aleatório de uma das situações experimentais controle $(\mathrm{CON})$ ou sprints (tiros de corrida em velocidade máxima) de 10 segundos (C10s). Em cada situação experimental foi realizada seis saltos em distância após a AC com 30 segundos de intervalo entre os saltos. $\mathrm{Na}$ situação $\mathrm{CON}$ os saltos foram realizados sem AC. Na situação C10s os voluntários realizaram dois sprints de 10 segundos com um minuto de intervalo entre os sprints. Os sprints foram realizados em uma superfície plana com pavimento de concreto. Os voluntários foram orientados a correr com máximo esforço possível. Após cinco minutos de repouso passivo, os voluntários realizados os saltos. A PSE foi registrada antes, imediatamente depois da $\mathrm{AC}$ e imediatamente antes dos saltos ( 5 min após a AC). A média das seis tentativas foi utilizado como a medida do desempenho no salto. O intervalo entre as situações experimentas foi de 48 horas. 


\section{Análise estatística}

As respostas individuais para todas as ACs foram avaliadas por meio do erro típico, seguindo o proposto por Healy \& Comyns(14) e Whelan et al.(15). Foram calculados a média e desvio padrão (DP) do desempenho nos seis saltos de cada voluntário em cada situação COM e C10s. O efeito das ACs foi considerado positivo (+) quando o desempenho do voluntário era 1,5 x DP maior que a média dos saltos da situação controle. Quando o desempenho do sujeito era 1,5 x DP menor que a média, o efeito da ACs foi considerado negativo (-) e, caso o desempenho nos arremessos estivessem entre $\pm 1,5 \times \mathrm{DP}$ da média, ele foi considerado não responsivo às ACs $(=)$ e apresentou-se as frequências absoluta e relativa (\%). A normalidade dos dados foi testada por Shapiro-Wilk. Foi utilizado o teste $t$ de Student pareado para comparar a distância do salto entre as situações controle e experimental. O nível de significância adotado foi menor que 5\%.

\section{Resultados}

A média da distância percorrida, após C10s, foi de $92( \pm 6) \mathrm{m}$ (Gráfico 1). A PSE antes, imediatamente e cinco minutos após a $\mathrm{AC}$ foram $6( \pm 1), \quad 14 \quad( \pm 2)$ e $10 \quad( \pm 2)$, respectivamente. A distância do salto foi maior na situação $\mathrm{C} 10 \mathrm{~s}(2,27 \pm 0,16)$ comparado ao controle $(2,20 \pm 0,20)$. A análise individual mostrou seis respostas positivas, três negativas e três indiferentes ao protocolo de potencialização.

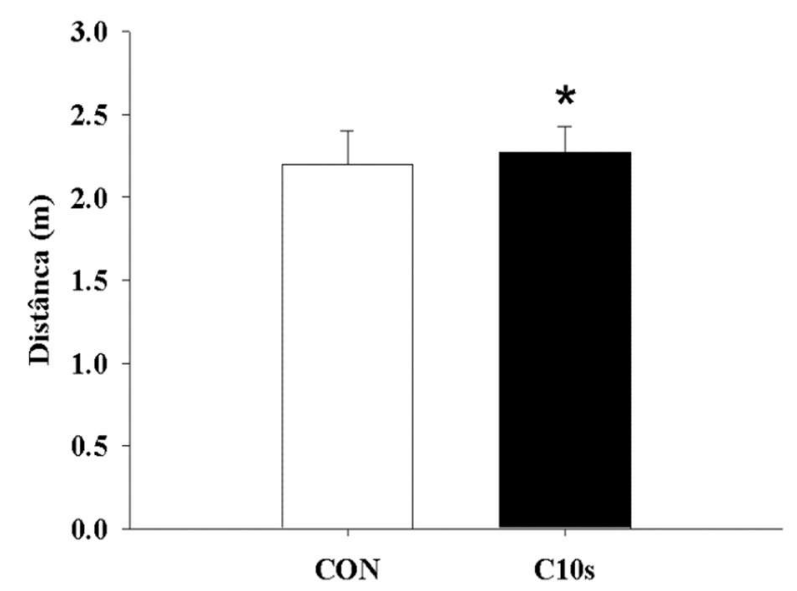

Gráfico 1 - Média e desvio padrão da distância do salto em distância entre as situações. Teste $t=-2.817 ; p=0.017 ; 0,67 ; 1$ $\beta=0,67 ; d=0,36\left(^{*}\right)$ maior que CON.
A Tabela 1 apresenta os resultados das respostas individuais classificadas quanto ao efeito dobre o desempenho e as análises descritivas das frequências absolutas e relativas. Observa-se que $50 \%$ dos participantes responderam positivamente ao protocolo da AC, $25 \%$ pioraram o desempenho do salto e em $25 \%$, a aplicação dos sprints não promoveu qualquer alteração.

Tabela 1 - Respostas individuais a atividade condicionante

\begin{tabular}{cc}
\hline \hline Voluntários & C10s \\
\hline 1 & $=$ \\
2 & + \\
3 & $=$ \\
4 & + \\
5 & - \\
6 & + \\
7 & + \\
8 & + \\
9 & $=$ \\
10 & + \\
11 & - \\
12 & - \\
Resposta positiva & $6(50 \%)$ \\
Resposta negativa & $3(25 \%)$ \\
Não responsivo & $3(25 \%)$ \\
\hline C10s: Classificação quanto ao efeito \\
observado comparando-se resultados em \\
C10s com resultados em CON.
\end{tabular}

\section{Discussão}

Os resultados do presente estudo mostraram que $50 \%$ dos participantes responderam positivamente ao protocolo da AC. Os demais ou não apresentaram nenhum efeito ou pioraram seu desempenho no salto em distância. As diferentes respostas à $\mathrm{AC}$ encontradas podem ser atribuídas a alguns fatores como fadiga, nível de força e estado de treinamento. De acordo com Rassier e Macintosh(5) o que determina o desempenho na atividade principal é o equilíbrio entre os mecanismos da PPA e os de fadiga. Apesar dos mecanismos serem semelhantes, o que diferencia se a AC causará fadiga ou potencialização é o tempo de recuperação entre a atividade de potencialização e a atividade principal. Se o intervalo for curto, a fadiga 
poderá predominar sobre os mecanismos de potencialização. Se o intervalo for longo demais, a fadiga é descartada, mas os mecanismos de potencialização também poderão ser dissipados. Alguns autores sugerem que, para intervalos menores que um minuto, a fadiga predominaria sobre os mecanismos de potenciação, diminuindo o desempenho, enquanto por períodos muito longos ( $>12 \mathrm{~min})$, a potenciação seria dissipada $(5,10,16)$.

A melhora do desempenho da atividade principal após a realização de uma $\mathrm{AC}$ é conhecida na literatura como PPA, na qual mecanismos centrais e musculares são previamente ativados em um nível ótimo a fim de potencializar o desempenho em determinado comportamento motor esportivo(1,2). Optou-se realizar análises das respostas individuais à $\mathrm{AC}$ por meio do erro típico porque, no ambiente competitivo de alto rendimento, análises individuais, muitas vezes, se fazem necessárias para melhor entendimento da resposta ao treinamento(15).

No presente estudo o intervalo entre as atividades foi de cinco minutos, na qual a PSE logo após a AC foi de $14( \pm 2)$ e antes da atividade principal foi de $10( \pm 2)$. A PSE é uma variável apontada como indicadora de ativação central ou pré-disposição à fadiga associada ao exercício(17). Assim, a diminuição dos valores de PSE cinco minutos após os sprints pode indicar um determinado nível de recuperação para alguns dos participantes. Tais achados, sugerem que o intervalo de cinco minutos entre as duas avaliações foi adequado para sobrepor os efeitos de PPA sobre os efeitos da fadiga nos participantes que melhoraram o desempenho no salto em distância.

Os resultados do presente estudo estão em linha com a literatura. Evetovich et al.(1) em estudo experimental, também, encontraram resultados positivos, negativos e nulos. De acordo com a revisão de Docherty et al.(4) o conhecimento para treinamentos complexos como os que envolvem PPA ainda carecem de mais investigações.

Outro fator que pode explicar as respostas individuais a AC é a predominância do tipo de unidade motora. Estudos anteriores mostraram maior potencialização em sujeitos com maior porcentagem de fibras do tipo II, sugerindo que esse tipo de unidade motora é mais susceptível à PPA(10). Infelizmente, o presente estudo não permite avaliar o efeito desta variável no desempenho do salto.

Outros fatores que podem modular os efeitos da potencialização são os tipos de unidades motoras recrutadas e o nível de treinamento(18). Estudos indicam que os mecanismos de potenciação são mais comuns em unidades motoras tipo IIx, também denominadas contração rápida, uma vez que essas unidades apresentam maior velocidade de condução de impulsos nervosos e melhor capacidade de fosforilação da miosina de cadeia leve em relação às unidades do tipo $\mathrm{I}(9)$. Esse mecanismo também explica por que indivíduos com experiência em treinamento de força e potência se beneficiam de estratégias de potenciação em comparação com indivíduos inexperientes, uma vez que o treinamento de força melhora o recrutamento de unidades do tipo II, especialmente unidades do tipo IIx(19). Contudo, o presente estudo mostrou que a PPA também é manifestada em sujeitos não treinados em força e sem experiência no salto, resultado que corrobora a melhora da distância do arremesso do peso em sujeitos não treinados e inexperientes após a realização do exercício de supino reto(11).

\section{Pontos fortes e limitações do estudo}

Acrescentado, parece não haver na literatura estudos que investigaram o efeito da realização de sprints no desempenho do salto em distância. $\mathrm{O}$ ponto forte do estudo foi evidenciar que os mecanismos de PPA também são manifestados em sujeitos não treinados e que a estratégia de utilização de $\mathrm{AC}$ não se aplica somente a atletas. Além disso, a pontencialização do salto em distância pode ser alcançada com uma única sessão de AC de sprints de curta duração.

Algumas limitações do presente estudo podem ser destacadas. Os resultados apresentados não permitem mensurar as contribuições centrais e periféricas da PPA. Dentro da proposta apresentada pelo estudo, foi possível apenas identificar os efeitos desses mecanismos. 


\section{Conclusão}

O presente estudo teve como objetivo avaliar o efeito de dois sprints de 10 segundos sobre desempenho do salto em distância em sujeitos não treinados e os resultados mostraram efeito positivo no desempenho do salto em distância após a realização do treinamento com sprints. Observa-se que $25 \%$ dos participantes piorou seu desempenho e que $25 \%$ não apresentou alteração. Assim, a utilização de sprints como AC deve ser prescrita de modo individualizado.

Estes achados indicam que ainda são necessárias mais investigações para melhor conhecer o efeito da PPA promovida pelo treinamento com sprints de 10 segundos sobre o desempenho no salto em distância.

\section{Declaração de conflito de interesses}

Não há conflito de interesses em relação ao presente trabalho.

\section{Declaração de financiamento}

Este estudo não foi financiado por qualquer agência ou por qualquer outra instituição pública ou privada.

\section{Referências}

1. Evetovich TK, Conley DS, Mccawley PF. Post-activation potentiation enhances upper and lower body athletic performance in collegiate men and women athletes. Journal of Strength and Conditional Research 2015;29(2):336-42. Available from: doi:10.1519/JSC.0000000000000728

2. Sale DG. Postactivation potentiation: Role in human performance. Exercise Sport Science Review 2002;30(3):138-143. Available from: doi:10.1136/bjsm.2002.003392

3. Rodriguez-Falces J, Duchateau J, Muraoka Y, Baudry S. M-wave potentiation after voluntary contractions of different durations and intensities in the tibialis anterior. Journal of Applied Physiology 2015;118(8):953-64. Available from: doi:10.1152/japplphysiol.01144.2014

4. Doherty D, Robbins D, Hodgson M. Complex training revisited: A review of its current status as a viable training approach.
Strength \& Conditioning Journal 2004;26(6):52-57.

5. Rassier D, Macintosh B. Coexistence of potentiation and fatigue in skeletal muscle. Brazilian Journal of Medical and Biological Research 2000;33(5):499-508. Available from: doi:10.1590/s0100879x2000000500003

6. HancocK AP. Sparks KE, Kullman EL. Postactivation potentiation enhances swim performance in collegiate swimmers. Journal of Strength and Conditional Research 2015;29(4):912-917. Available from:

doi:10.1519/JSC.0000000000000744.

7. Turner AP, Bellhouse S, Kilduff LP, Russell M. Postactivation potentiation of sprint acceleration performance using plyometric exercise. Journal of Strength and Conditional Research 2015;29(2):343$350 . \quad$ Available from: doi:10.1519/JSC.0000000000000647

8. Sarramian VG, Turner AN, Greenhalgh AK. Effect of postactivation potentiation on fifty-meter freestyle in national swimmers. Journal of Strength and Conditional Research 2015;29(4):1003-1009. Available from:

doi:10.1519/JSC.0000000000000708.

9. Chiu LZF, Fry AC, Weiss LW, Schilling BK, Brown LE, and Smith SL. Potentiation of Vertical Jump performance During a Snatch Pull Exercise Session. Journal of Applied Biomechanics 2012; 28:627-635. Available from: doi:10.1123/jab.28.6.627 .

10. Xenofondos A, Laparidis K, Kyranoudis A, Galazoulas Ch, Bassa E, Kotzamanidis C. Post-activation potentiation: factors affecting it and the effect on Performance. Journal of Physician Education and Sport 2010;28(3):32-38.

11. Borba DA, Ferreira-Júnior JB, Ramos MVD, Gomes RLD, Guimarães JB, Oliveira JRV. Bench press exercise performed as conditioning activity improves shot put performance in untrained subjects. Motriz 2018;24(4):1-5. Available from: doi:10.1590/S1980- 
12. Jackon AS. Pollock ML Generalized equations for predicting body density of men. British Journal of Nutrition 1978;409(3):497-504. Available from: 10.1079/BJN19780152

13. Borg GAV, Noble BJ. Perceived exertion. In: Wilmore JH, editor. Exercise and Sport Sciences Reviews. Vol. 2. Academic Press, p. 131-53, New York, 1974.

14. Heal R, Comyns TM. The application of postactivation potentiation methods to improve sprint speed. Journal of Strength and Conditional Research 2017;39(1): 1-9. Available from: 10.1519/SSC.0000000000000276

15. Whelan N, O'Regan C, Harrison AJ. Resisted sprints do not acutely enhance sprinting performance. Journal of Strength and Conditional Research 2014;28(7):1858-66. Available from: doi: 10.1519/JSC.0000000000000357

16. Kilduff LP, Bevan HR, Kingsley MI, Owen NJ, Bennett MA, Bunce PJ, et al. Postactivation potentiation in professional rugby players: optimal recovery. Journal of Strength and Conditional Research 2007;21(4):1134-1138. Available from: doi:10.1519/R-20996.1

17. Siqueira OD et al. A utilização da PSE como indicadora de intensidade de um teste progressivo de corrida intermitente em jogadores de futebol. Coleção Pesquisa em Educação Física 2011;10;(5):7-14.

18. Gourgoulis V, Aggeloussis N, Kasimatis P, Mavromatis G, Garas A. Effect of a submaximal half-squats warm-up program on vertical jumping ability Journal of Strength and Conditional Research 2003;17:342-344. Available from: doi:10.1519/1533-4287(2003)017

19. Balsalobre-Fernández $\mathrm{C}$, Tejero-González CM, Campo-Vecino J, Alonso-Curiel D. The Effects of a Maximal Power Training Cycle on the Strength, Maximum Power, Vertical Jump Height and Acceleration of High-Level 400-Meter Hurdlers. Journal of Human Kinetics 2013;36:119-26. Available from: doi:10.2478/hukin-2013-0012 